\title{
Removal of lead ion from aqueous solution by Metal Organic Framework
}

\author{
Hossein Shayegan,Yeganeh Davoudabadi Farahani, Vahid Safarifard* \\ Department of Chemistry, Iran University of Science and Technology, Tehran 16846-13114, Iran. \\ Email: vsafarifard@iust.ac.ir; Fax: +98 21 73021415; Tel: +98 2173228327
}

\begin{abstract}
:
Since $\mathrm{Pb}$ (II) is one of the toxic water contamination agents, finding an efficient way to remove it from wastewater has become a serious issue in the world. Herein, a metal-organic framework, formulated as (TMU-23), with amide-decorated pores was prepared to investigate its potential in $\mathrm{Pb}$ (II) removal performance. At first, various determining factors including $\mathrm{pH}$ and adsorbent dosage were optimized. Then, the MOF adsorption capacity was measured about $434.7 \mathrm{mg}^{-1} \mathrm{~g}^{-1}$. Moreover, reusability feature and effect of competitive cations were checked. Adsorptiondesorption tests showed that during three cycles, the MOF could act with favorable efficiency. Modeling calculations illustrate that pseudo-second-order and Langmuir models provide the best description for adsorption mode. Based on these models, monolayer adsorption behavior was responsible for adsorption process through chemical interaction between the analyte and the walls of MOF. Also, the possible adsorption mechanism was examined by exploiting of FTIR spectroscopy, EDS and SEM images. The significant affinity between amide groups of pillars and $\mathrm{Pb}(\mathrm{II})$ could cause good removal performance.
\end{abstract}

Keywords: Heavy metal ions; Metal-organic frameworks; Adsorption; Langmuir; Freundlich.

\section{Introduction:}

Recently, metal-organic frameworks, abbreviated as MOFs, have attracted attention worldwide according to their unique features including highly ordered crystalline structure [1], abundant active surface sites [2], tunable pore size [3], high surface area [4] and so forth. MOFs are built from metal-containing units [secondary building units (SBUs)] or metal ions/clusters and organic linkers through making strong bonds [5]. In numerous studies, researchers have examined a wide range of MOFs' applications including gas storage and separation, molecular recognition, liquid sorption, catalysis and capture of heavy metal ions.

Water contamination agents containing toxic and harmful chemicals have been threatened the human and environmental health [6,7]. Unfortunately, the development of industry has produced tremendous amounts of wastewater containing heavy metals ions (e.g. $\mathrm{Cr}^{3+}, \mathrm{Ni}^{2+}, \mathrm{Co}^{2+}, \mathrm{Mn}^{2+}$, and 
$\left.\mathrm{Pb}^{2+}\right)$. These toxic heavy metals, especially lead(II), is categorized among the non-essential trace elements for biological systems with the maximum permissible levels of 0.015 and $0.01 \mathrm{mg} \mathrm{L}^{-1}$ in potable water based on the Environmental Protection Agency (EPA) and World Health Organization (WHO), respectively $[8,9] . \mathrm{Pb}$ (II) ions release in the environment as a result of radiation device, lead mining, water pipe, battery recycling plants, submarine cable sheathing, electronics assembly plants, solar cell, ceramic, urban rainwater runoff, and paint industries as well as whitening products [10]. Excessive intake of lead(II) will lead to serious illnesses such as lung, kidney, and skin cancers, the nervous and digestive systems damages [11], liver failure, anaemia, nephritis and renal disorders even at a very low concentration. As water is the main carrier of lead ions to human bodies, the decontamination of this cation contaminated wastewater from industrial establishments has become a major challenge. Membrane filtration, chemical precipitation, flocculation, reverse osmosis, adsorption, etc. are some of the routes for removing lead(II) from water. Among these methods and technologies, adsorption is known as a favorable candidate for the purification of wastewater due to environmental friendliness, cost-effectiveness and easy operation. Various adsorbents have been introduced such as active carbon and its derivations, metallogels, LDHs, and polymers [12]. But some disadvantages like complicated preparation, slow adsorption kinetics, difficult regeneration as well as poor efficiency restrict their performance.

\section{Experimental section:}

Synthesis of bpfb:

1,4-phenylenediamine $(1.081 \mathrm{~g} ; 10 \mathrm{mmol})$ was dissolved in $50 \mathrm{ml}$ of dry THF containing $2.84 \mathrm{ml}$ of TEA (20.4 mmol). Then, isonicotinoyl chloride hydrochloride $(3.560 \mathrm{~g}, 20 \mathrm{mmol})$ was eked into these solutions and heated under reflux for $24 \mathrm{~h}$. The final suspension was filtered, dried under ambient conditions, and poured into an aqueous saturated solution of $\mathrm{Na}_{2} \mathrm{CO}_{3}(50 \mathrm{ml})$. The obtained white solid was finally filtered and dried and consumed as the MOF's pillar.

Synthesis of (TMU-23):

$\mathrm{Zn}\left(\mathrm{NO}_{3}\right)_{2} .6 \mathrm{H}_{2} \mathrm{O}(0.297 \mathrm{~g}, 1 \mathrm{mmol}), \mathrm{H}_{2} \mathrm{oba}(0.258 \mathrm{~g}, 1 \mathrm{mmol})$, and bpfb $(0.5 \mathrm{mmol})$ were dissolved in DMF $(50 \mathrm{~mL})$ together, then the mixture was sonicated until the reaction was completed ( 30 min) and Red-brown (TMU-23) powders were obtained as pure phases. In the next step, it was washed by DMF, and dried at room temperature as reported in the literature previously [13].

Adsorption Experiments:

In our work, the impact of various parameters on the absorption proficiency of TMU-23 was examined. In this way, $50 \mathrm{ml}$ of lead solution was added to $5 \mathrm{mg}$ of TMU-23 at room temperature and in ultrasonic baths. $\mathrm{Pb}\left(\mathrm{NO}_{3}\right)_{2}$ were utilized as the source of lead ions. The solution $\mathrm{pH}$ was controlled via addition of $0.1 \mathrm{ml}$ of $0.1 \mathrm{M}$ sodium hydroxide or $0.1 \mathrm{M} \mathrm{HCI}$. After that, the final mixture was centrifuged for 6 minutes. Finally, sampling from the solution was done.

\section{Result and Discussion:}


The PXRD patterns of the as-prepared framework conformed to the simulated (derived from the single crystal structures) PXRD patterns (Fig. 1a). Also, Fourier transformed infrared spectroscopy confirmed the correction of the reported synthesis (Fig. 1b).
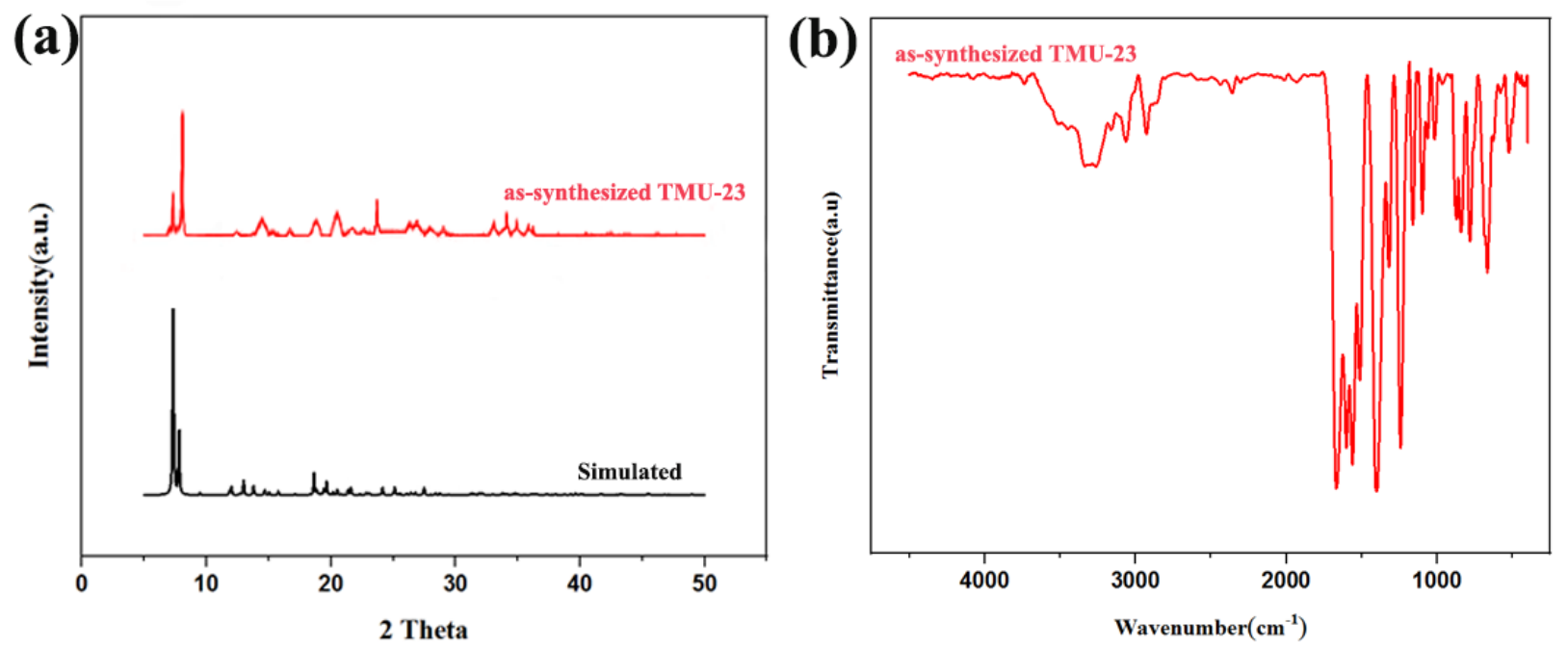

Figure 1. (a) PXRD of as-synthesized (red) and simulated (black) TMU-23. (b) FT-IR spectra of TMU-23.

$\mathbf{P b}$ (II) Adsorption Studies. Ultrasonic-assisted adsorption of Lead(II) from aqueous solutions were examined by using TMU-23 in order to find the optimal ions initial concentration, $\mathrm{pH}$, contact time, and adsorbent dosage. All experiments were done at room temperature at different conditions, based on the designed experiments.

Effect of $\mathbf{p H}$. Absorption procedure relies on the $\mathrm{pH}$ strongly. First, $50 \mathrm{ppm}$ of $\mathrm{Pb}$ (II) solution was made to examine the absorption behavior of TMU-23. $5 \mathrm{mg}$ of the framework was added to $50 \mathrm{ml}$ of this solution in separate containers. By increasing the $\mathrm{pH}$ of the solution, the amount of $\mathrm{H}^{+}$ion declines in the solution and at $\mathrm{pH}=7$, the max amount of lead(II) was adsorbed (Fig. 2).

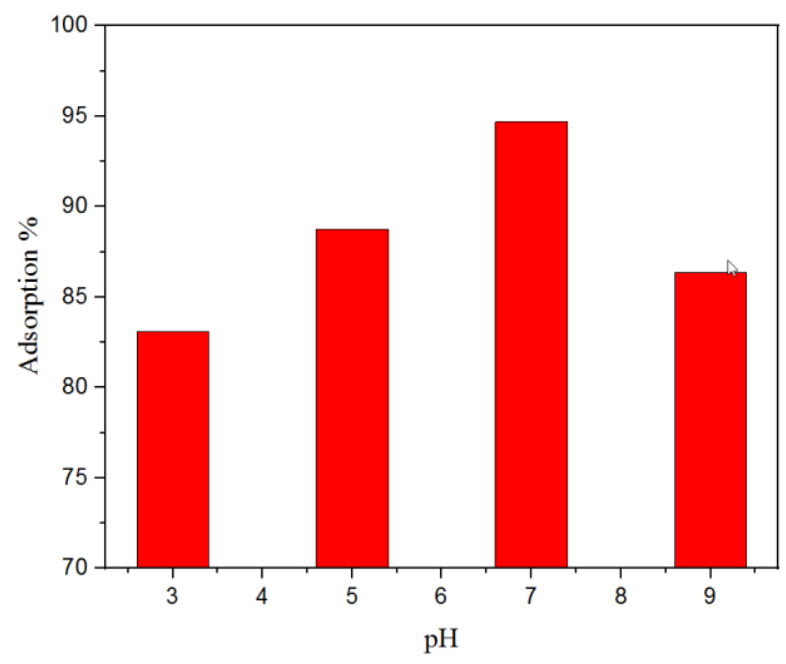


Figure 2. $\mathrm{pH}$ solution effect on $\mathrm{Pb}^{2+}$ ions adsorption efficiency for TMU-23.

Study of Sorption Kinetics. To realize the mechanism of the adsorption process, various adsorption models were proposed in literature containing intraparticle diffusion, pseudo-first-order and pseudo-second-order models determining the types of adsorption. TMU-23 adsorption performance followed pseudo-first-order model with a high value of correlation coefficient $\left(\mathrm{R}^{2}\right.$ $=0.994)$ (Fig. 3a). Data shows that the chemical adsorption might control the adsorption process of $\mathrm{Pb}$ (II) with a more satisfactory correlation compared to the pseudo-first-order model $\left(\mathrm{R}^{2}=0.988\right)$ (Fig. 3b).
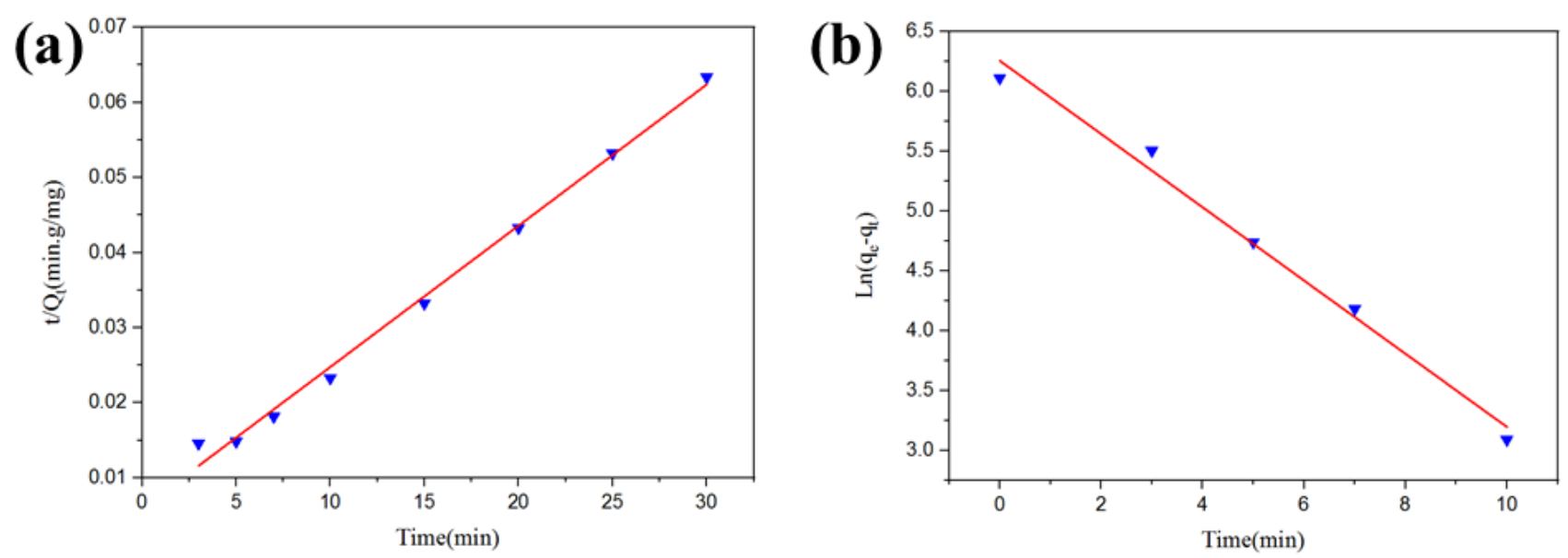

Figure 3. (a) Pseudo-second-order. (b) Pseudo-First-order for adsorption $\mathrm{Pb}^{2+}$ ions TMU-23.

Investigation of Comparative Adsorption. In order to investigate the effect of comparative ions on TMU-23 performance, common heavy metals including $\mathrm{Co}^{2+}, \mathrm{Hg}^{2+}, \mathrm{Cd}^{2+}, \mathrm{Al}^{3+}, \mathrm{As}^{3+}, \mathrm{Ni}^{2+}$, $\mathrm{Cu}^{2+}, \mathrm{Pb}^{2+}$ and $\mathrm{Cr}^{3+}$ metal cations were selected. As indicated in Fig. 4, our proposal framework adsorbs $\mathrm{Pb}(\mathrm{II})$ more efficiently compared to others.

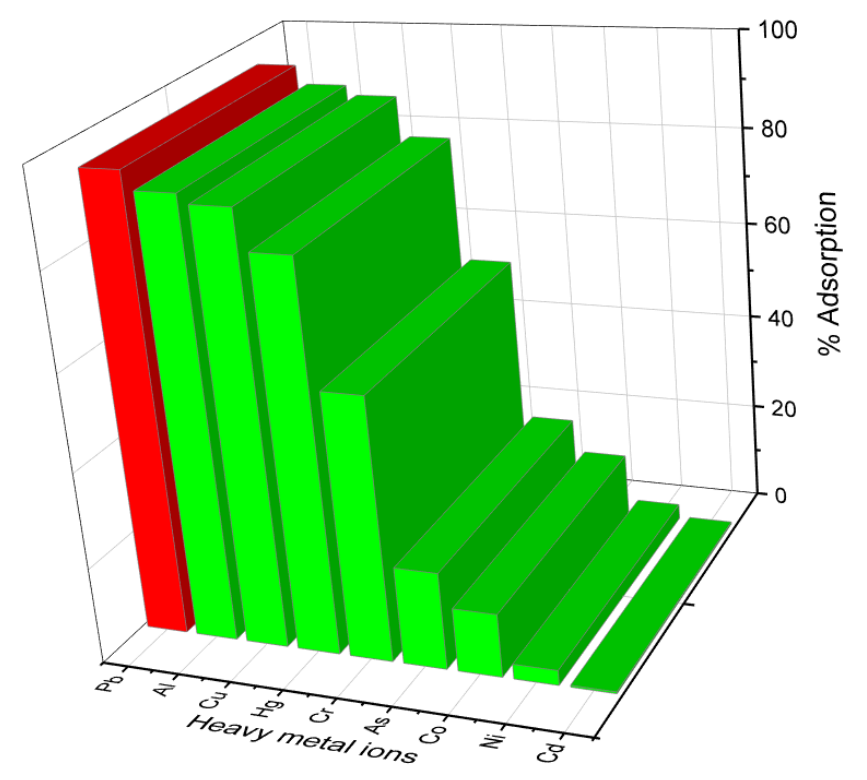


Figure 4. Comparative adsorption of different heavy-metal ions.

Reusability Study. To check the recyclability of TMU-23, three adsorption-desorption cycles were carried out. The obtained results were collected in Fig. 5. Desorption process was established by adding $2 \mathrm{~mL}$ of deionized water to the material, then the solution was stirred for 20 minutes at room temperature. The amounts of total lead(II) were determined by ICP-AES. The percentage of adsorption after three cycles confirmed the reusability of this adsorbent during these adsorptiondesorption cycles.

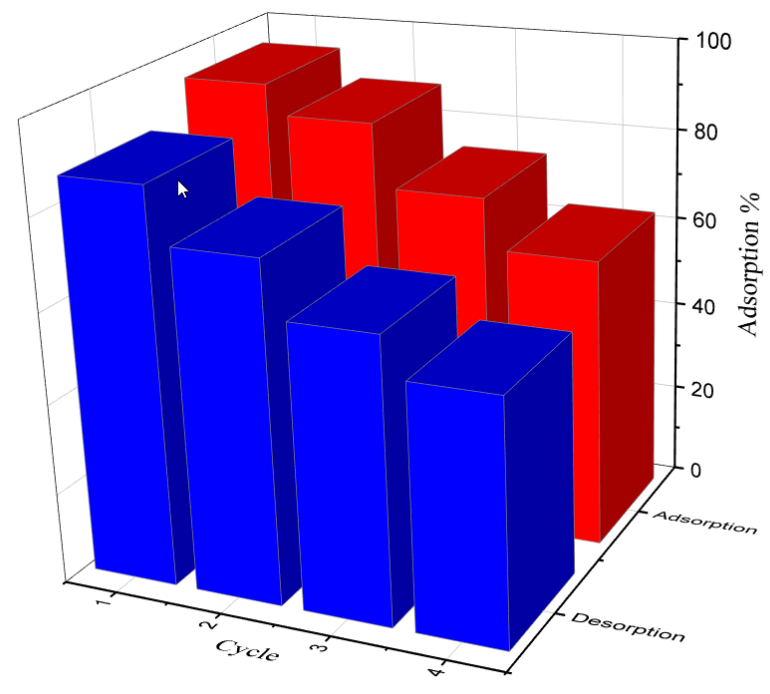

Figure 5. Adsorption-desorption cycles for TMU-23.

\section{Conclusion}

In the present study, a metal-organic framework, namely TMU-23, with amide-decorated channels were prepared via utilizing $\mathrm{H}_{2} \mathrm{Oba}$ and bpfb as linkers to check the adsorption performance toward various heavy metal ions. The collected data illustrated that this MOF uptake $\mathrm{Pb}$ (II) more efficiently compared to other metal ions. Then, the effects of $\mathrm{pH}$, contact time and adsorbent dosage on adsorption performance of the proposed material were investigated. The adsorption process achieved to the equilibrium less than $15 \mathrm{~min}$ which a favorable time for these kinds of adsorbents. At the end of the paper, by using different analyses, including FTIR, EDS, PXRD and SEM images, the mechanism of sorption was presented. We showed that the analyte was adsorbed on the surface of the MOF structure through monolayer chemical interaction.

\section{Reference :}

[1] C. Yu, X. Han, Z. Shao, L. Liu, H. Hou, High Efficiency and Fast Removal of Trace Pb (II) from Aqueous Solution by Carbomethoxy-Functionalized Metal-Organic Framework, Crystal Growth \& Design, 18 (2018) 1474-1482. 
[2] J. Chen, K. Liu, M. Jiang, J. Han, M. Liu, C. Wang, C. Li, Controllable preparation of porous hollow carbon sphere@ ZIF-8: Novel core-shell nanomaterial for Pb2+ adsorption, Colloids and Surfaces A: Physicochemical and Engineering Aspects, 568 (2019) 461-469.

[3] R. Zhang, Y. Liu, Y. An, Z. Wang, P. Wang, Z. Zheng, X. Qin, X. Zhang, Y. Dai, B. Huang, A waterstable triazine-based metal-organic framework as an efficient adsorbent of $\mathrm{Pb}$ (II) ions, Colloids and Surfaces A: Physicochemical and Engineering Aspects, 560 (2019) 315-322.

[4] Z.S. Hasankola, R. Rahimi, V. Safarifard, Rapid and efficient ultrasonic-assisted removal of lead (II) in water using two copper-and zinc-based metal-organic frameworks, Inorganic Chemistry Communications, 107 (2019) 107474.

[5] S. Kazemi, V. Safarifard, Carbon dioxide capture in MOFs: The effect of ligand functionalization, Polyhedron, 154 (2018) 236-251.

[6] J. Wang, Y. Li, Z. Lv, Y. Xie, J. Shu, A. Alsaedi, T. Hayat, C. Chen, Exploration of the adsorption performance and mechanism of zeolitic imidazolate framework-8@ graphene oxide for $\mathrm{Pb}$ (II) and 1naphthylamine from aqueous solution, Journal of colloid and interface science, 542 (2019) 410-420.

[7] A.A. Alqadami, M.A. Khan, M.R. Siddiqui, Z.A. Alothman, Development of citric anhydride anchored mesoporous MOF through post synthesis modification to sequester potentially toxic lead (II) from water, Microporous and Mesoporous Materials, 261 (2018) 198-206.

[8] H. Zhu, J. Yuan, X. Tan, W. Zhang, M. Fang, X. Wang, Efficient removal of Pb 2+ by Tb-MOFs: identifying the adsorption mechanism through experimental and theoretical investigations, Environmental Science: Nano, 6 (2019) 261-272.

[9] C. Yu, Z. Shao, H. Hou, A functionalized metal-organic framework decorated with $\mathrm{O}-$ groups showing excellent performance for lead (II) removal from aqueous solution, Chemical science, 8 (2017) 7611-7619. [10] D. Chen, W. Shen, S. Wu, C. Chen, X. Luo, L. Guo, Ion exchange induced removal of Pb (II) by MOFderived magnetic inorganic sorbents, Nanoscale, 8 (2016) 7172-7179.

[11] Y. Wu, Y. Ma, G. Xu, T. Xia, W. Liu, Z. Dong, Q. Yuan, C. Zhang, Q. Hu, Synthesis of two novel H 4 TCPBDA-based metal-organic frameworks and their application in lead ion adsorption, Journal of Materials Science, 54 (2019) 2093-2101.

[12] M. Lu, L. Li, S. Shen, D. Chen, W. Han, Highly efficient removal of Pb 2+ by a sandwich structure of metal-organic framework/GO composite with enhanced stability, New Journal of Chemistry, 43 (2019) 1032-1037.

[13] V. Safarifard, S. Rodríguez-Hermida, V. Guillerm, I. Imaz, M. Bigdeli, A. Azhdari Tehrani, J. Juanhuix, A. Morsali, M.E. Casco, J. Silvestre-Albero, Influence of the amide groups in the CO2/N2 selectivity of a series of isoreticular, interpenetrated metal-organic frameworks, Crystal Growth \& Design, 16 (2016) 6016-6023. 